\title{
Photonic superdiffusive motion in resonance radiation trapping
}

\author{
M. N. Berberan-Santos ${ }^{\text {a) }}$ \\ Centro de Química-Física Molecular, Instituto Superior Técnico, 1049-001 Lisboa, Portugal \\ E. J. Nunes-Pereira \\ Centro de Física, Escola de Ciências, Universidade do Minho, 4710-057 Braga, Portugal \\ J. M. G. Martinho \\ Centro de Química-Física Molecular, Instituto Superior Técnico, 1049-001 Lisboa, Portugal
}

(Received 15 February 2006; accepted 18 July 2006; published online 7 November 2006)

In this work we consider the relation between the jump length probability density function and the line shape function in resonance radiation trapping in atomic vapors. The two-sided jump length probability density function suitable for a unidimensional formulation of radiative transfer is also derived. As a side result, a procedure to obtain the Maxwell distribution of velocities from the Maxwell-Boltzmann distribution of speeds was obtained. General relations that give the asymptotic jump length behavior and the Lévy flight parameter $\mu$ for any line shape are obtained. The results are applied to generalized Doppler, generalized Lorentz, and Voigt line shape functions. It is concluded that the lighter the tail of the line shape function, the less heavy the tail of the jump length probability density function, although this tail is always heavy, with $\mu \leqslant 1$. (C) 2006 American Institute of Physics. [DOI: 10.1063/1.2364889]

\section{INTRODUCTION}

The convectionless, random motion of a particle in isotropic, homogeneous three-dimensional space is in most cases governed by the diffusion equation, ${ }^{1-5}$

$$
\frac{\partial p(\mathbf{r}, t)}{\partial t}=D \nabla^{2} p(\mathbf{r}, t)
$$

where $D$ is the diffusion coefficient of the particle in the medium and $p(\mathbf{r}, t)$ is the probability density function (PDF) for the position of the particle. Usually the particle in question is a material one (atom, molecule, etc.) and the motion is thermal in origin. However, the diffusive motion, properly defined, also applies to photons, as will be discussed here.

The diffusion equation, once believed to be of universal validity, can be obtained from a more fundamental relation, a distribution of jump lengths and waiting times, ${ }^{4} f(r, t)$, provided some conditions hold. If jump length and waiting time are independent, a PDF of jump lengths $f(r)$ and a PDF of waiting times between jumps, $g(t)$, can be defined. For Eq. (1) to hold, the diffusion coefficient $D$ must be finite. It can be written as

$$
D=\frac{\left\langle r^{2}\right\rangle}{6 \tau}
$$

where $\tau$ is the average waiting time between jumps, assumed here to be finite, and

$$
\left\langle r^{2}\right\rangle=\int_{0}^{\infty} r^{2} f(r) d r
$$

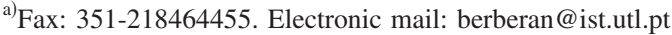

However, Brownian-type (normal) diffusion is not the only possibility for particle motion. ${ }^{2-6}$ For instance, the second moment of $f(r)$ may a priori be infinite. This at first physically strange result has indeed been identified in a few cases. $^{2-8}$ When it happens the diffusion coefficient is also infinite and the diffusion equation does not hold. One then has a special type of anomalous diffusion, called superdiffusion or hyperdiffusion. In such a case $f(r)$ decays very slowly when $r \rightarrow \infty$ (it is said to be heavy tailed or "fat" tailed), the particle's motion is a Lévy flight, and its trajectory defines a fractal. ${ }^{2-5,8}$ The appropriate generalization of the diffusion equation in an infinite, isotropic threedimensional space is an integrodifferential equation,

$$
\frac{\partial p(\mathbf{r}, t)}{\partial t}=\frac{1}{\tau}\left[\int f\left(\mathbf{r}, \mathbf{r}^{\prime}\right) p\left(\mathbf{r}^{\prime}, t\right) d \mathbf{r}^{\prime}-p(\mathbf{r}, t)\right],
$$

where $f\left(\mathbf{r}, \mathbf{r}^{\prime}\right)$ is the average probability that a jumping particle initially at point $\mathbf{r}^{\prime}$ reaches the volume element around point $\mathbf{r}$ in one jump. For isotropic media, $f\left(\mathbf{r}, \mathbf{r}^{\prime}\right)$ should only depend on distance. Integration of this probability over all directions then gives $f\left(\left|\mathbf{r}-\mathbf{r}^{\prime}\right|\right)=f(r)$. Equation (4) is a particular form of the so-called master equation for stochastic processes, ${ }^{1}$ it being implicit that the transit time from point $\mathbf{r}^{\prime}$ to point $\mathbf{r}$ is negligible. The concept of Lévy walk ${ }^{4,5}$ has been introduced for cases where the transit time is finite, and is usually a function of the distance traveled. The problem of radiation trapping should also be formulated as a Lévy walk, whenever the propagation time of the photons becomes significant in comparison with the excited state lifetimes.

For resonance radiative trapping in atomic vapors, the relevant particles are quanta. The photons are absorbed and reemitted many times in the medium, changing their frequency in the process. For this reason, the motion of the photons does not obey a diffusion equation, and is conve- 
niently described instead by the above integrodifferential equation, called in this context the Holstein-Biberman equation. ${ }^{9-11}$ In the case of complete frequency redistribution, i.e., when the frequency of the emitted photon is independent of the frequency of the previously absorbed photon, the jump length PDF can be written as ${ }^{8}$

$$
f(r)=\int_{-\infty}^{\infty} \Phi(x)^{2} e^{-\Phi(x) r} d x,
$$

where $r$ is a dimensionless distance, $x$ is a renormalized frequency, and $\Phi(x)$ is the spectral line shape function (normalized absorption and emission spectra, so that $\left.\int_{-\infty}^{+\infty} \Phi(x) d x=1\right)$ of the resonance line in question. Complete frequency redistribution applies whenever the collision frequency is higher than the emission rate, so that the momentum of an emitting atom is uncorrelated with the momentum it had previously while absorbing the exciting photon. This is the case in most laboratory experiments, where the propagation time of the photons is also negligible in comparison with the excited state lifetimes.

Superdiffusivity is a consequence of the heavy-tailed nature of $f(r)$. The flight of the particles is superdiffusive whenever for large $r$,

$$
f(r) \sim r^{-(1+\mu)},
$$

with $\mu \leqslant 2 .^{2-6}$ This parameter must also obey $\mu>0$ owing to the normalization of $f(r)$. Note that for $\mu \leqslant 1$ the first moment $\langle r\rangle$ is also infinite. One-sided (i.e., defined for $r \geqslant 0$ only) Lévy distributions imply ${ }^{2} \mu \leqslant 1$.

A powerful alternative formulation of the diffusion problem that encompasses the ordinary diffusion equation [Eq. (1)] is the bifractional diffusion equation, which contains a generalized diffusion coefficient and can be solved by the methods of fractional calculus. ${ }^{4-6}$ For the present case, in particular, it follows that the characteristic distance traveled, $r_{c}(t)$, appropriately defined in order to remain finite, ${ }^{4,5}$ scales with time as ${ }^{4-6}$

$$
r_{c}(t) \sim t^{1 / \mu}
$$

For normal diffusion $\mu=2$.

It was previously argued ${ }^{7}$ and later demonstrated ${ }^{8}$ that the motion of the photons was superdiffusive in the case of Doppler (or Gaussian) and Lorentz (or Cauchy) spectral line shapes,

$$
\begin{aligned}
& \Phi_{D}(x)=\frac{1}{\sqrt{\pi}} e^{-x^{2}}, \\
& \Phi_{L}(x)=\frac{1}{\pi} \frac{1}{1+x^{2}} .
\end{aligned}
$$

The respective Lévy parameters, obtained by means of ad hoc arguments, ${ }^{8}$ are $\mu=1$ and $\mu=\frac{1}{2}$. In this way, the Doppler line shape corresponds to the so-called ballistic case, ${ }^{4} r_{c}(t)$ $\sim t$, and the Lorentz line shape leads to an extreme case of superdiffusive behavior, with $r_{c}(t) \sim t^{2}$.

In this work we introduce a distribution of effective opacities $H(k)$ (Sec. II) and then derive general relations that give the asymptotic behavior of the one-sided jump length
PDF $f(r)$ and the Lévy parameter $\mu$ for any given line shape $\Phi(x)$ (Sec. III). The moments of the jump length PDF are evaluated in Sec. IV. The two-sided jump length PDF $g(z)$ along an arbitrary axis is next derived (Sec. V). Finally, the results are applied to generalized Doppler, generalized Lorentz, and Voigt line shape functions (Sec. VI). The main conclusions are summarized in Sec. VII.

\section{DISTRIBUTION OF EFFECTIVE LINE OPACITIES}

The jump length PDF in Eq. (5) is written in terms of the dimensionless distance $r$, given by $r=n \sigma_{0} l / \Phi(0)$, where $n$ is the number density of particles, assumed to be homogeneously distributed, $\sigma_{0}$ is the particle's center-of-line absorption cross section, and $l$ is the path length. Parameter $r$ is therefore proportional to the center-of-line opacity $k_{0}$ (also called center-of-line optical depth), which is given by $k_{0}$ $=n \sigma_{0} l$. The opacity for any other frequency is given by $k(x)=n \sigma_{0} \Phi(x) l$. The dimensionless distance used here needs to be translated into physical distances for actual systems and it is therefore useful to estimate orders of magnitude for the quantities involved. Number densities for neutral vapors and plasmas fall approximately in the range of $10^{8}-10^{18} \mathrm{~cm}^{-3}$. Using a resonance center-of-line absorption cross section of $10^{-13} \mathrm{~cm}^{2}$ and for a number density of $10^{16} \mathrm{~cm}^{-3}$, dimensionless distances between 0.1 and $10^{3}$ correspond to path lengths from $0.5 \mu \mathrm{m}$ to $5 \mathrm{~mm}$. On the other hand, considering an average value of $10^{5} \mathrm{~cm}^{-3}$ for interstellar space conditions and using an absorption cross section of $10^{-9} \mathrm{~cm}^{2}$, the $0.1-10^{3}$ range of dimensionless distances now translates into path lengths from $5 \mathrm{~m}$ to $50 \mathrm{~km}$.

It is convenient to rewrite Eq. (5) in the following form:

$$
f(r)=\int_{0}^{\infty} H(k)\left(k e^{-k r}\right) d k,
$$

where $H(k)$ is a PDF of effective line opacities. When the effective opacity distribution is a delta function, it follows from Eq. (10) that the jump length PDF is exponential. This is the case for monochromatic radiation. However, real spectral line shape functions imply the existence of photons with a wide range of frequencies, hence broad distributions of opacities.

In the general case, the $H(k)$ PDF can be related to the line shape $\Phi(x)$ function by means of Eq. (5). In fact, it follows from Eq. (10) that $k H(k)$ is the inverse Laplace transform of $f(r)$, given in turn by Eq. (5). Application of the real inversion formula for the Laplace transform (with parameter $c=0){ }^{12}$

$$
\begin{aligned}
k H(k)= & \frac{1}{\pi} \int_{0}^{\infty}[\operatorname{Re}[f(i \omega)] \cos (k \omega) \\
& -\operatorname{Im}[f(i \omega)] \sin (k \omega)] d \omega,
\end{aligned}
$$

gives

$$
H(k)=\frac{1}{k} \int_{-\infty}^{\infty} \Phi(x)^{2} \delta[k-\Phi(x)] d x,
$$

and, assuming a symmetric line shape, 


$$
H(k)=\frac{2}{k} \int_{0}^{\infty} \Phi(x)^{2} \delta[k-\Phi(x)] d x .
$$

Making the change of variable $y=\Phi(x)$, Eq. (13) becomes

$$
H(k)=-\frac{2}{k} \int_{0}^{\Phi(0)} y^{2} \Psi^{\prime}(y) \delta(y-k) d y,
$$

it being assumed that $\Phi(x)$ is nonzero for all $x$, and where $\Psi(y)$ is the inverse function of $\Phi(x), \Psi(y)=\Phi^{-1}(y)$. Equation (14) can be rewritten as

$$
H(k)= \begin{cases}-2 k \Psi^{\prime}(k) & \text { if } k<\Phi(0) \\ 0 & \text { if } k \geqslant \Phi(0) .\end{cases}
$$

This equation explicitly relates the distribution of effective opacities with the line shape function. It will be next used (Sec. III) to study the asymptotic behavior of the jump length PDF and will be applied in Sec. VI to several specific line shapes.

\section{ASYMPTOTIC BEHAVIOR OF THE JUMP LENGTH PDF}

Using Eq. (15), Eq. (5) becomes

$$
f(r)=-2 \int_{0}^{\Phi(0)} k^{2} \Psi^{\prime}(k) e^{-k r} d k .
$$

In order to obtain the asymptotic behavior of $f(r)$ for large $r$, it is convenient to perform the change of variable $y=k r$,

$$
f(r)=-2 r^{-3} \int_{0}^{\Phi(0) r} y^{2} \Psi^{\prime}(y / r) e^{-y} d y .
$$

In this way, the asymptotic behavior of $f(r)$ for large $r$ is

$$
f(r) \sim r^{-3} \int_{0}^{\infty} y^{2} \Psi^{\prime}(y / r) e^{-y} d y .
$$

Integration by parts of Eq. (16) leads to

$$
f(r)=2 \int_{0}^{\Phi(0)}(2-k r) k \Psi(k) e^{-k r} d k,
$$

and performing the same change of variable $y=k r$,

$$
f(r)=2 r^{-2} \int_{0}^{\Phi(0) r}(2-y) y \Psi(y / r) e^{-y} d y,
$$

hence an alternative form for the asymptotic behavior of $f(r)$ for large $r$ is

$$
f(r) \sim r^{-2} \int_{0}^{\infty}(2-y) y \Psi(y / r) e^{-y} d y .
$$

If the line shape function has a power-law asymptotic behavior for large $x$ (the "wings"),

$$
\Phi(x) \sim x^{-p} \quad(p>1),
$$

then the inverse function goes as

$$
\Psi(k) \sim k^{-1 / p},
$$

for small $k$, and Eq. (18) becomes

$$
f(r) \sim r^{1 / p-2} \int_{0}^{\infty} y^{1-1 / p} e^{-y} d y=\Gamma(2-1 / p) r^{1 / p-2},
$$

and therefore

$$
f(r) \sim r^{1 / p-2},
$$

i.e., the parameter $\mu$ of Eq. (6) is

$$
\mu=1-\frac{1}{p} \quad(p>1) .
$$

\section{MOMENTS OF THE JUMP LENGTH PDF}

The moments of $f(r)$ can be computed using the $H(k)$ PDF,

$$
\left\langle r^{n}\right\rangle=\int_{0}^{\infty} r^{n} f(r) d r=n ! \int_{0}^{\infty} \frac{H(k)}{k^{n}} d k=n !\left\langle k^{-n}\right\rangle,
$$

as results from Eq. (10). Using Eq. (13), it follows from Eq. (27) that

$$
\langle r\rangle=\int_{0}^{\infty} \frac{H(k)}{k} d k=2 \int_{0}^{\infty} d k \rightarrow \infty,
$$

implying that $\mu \leqslant 1$, independently of the line shape function, ${ }^{8}$ as long as $\Phi(x)$ is nonzero everywhere. Equation (5) may thus be viewed as a special integral transform that converts any two-sided nonzero line shape function $\Phi(x)$ into a one-sided PDF with all moments infinite, i.e., with a heavy tail.

\section{TWO-SIDED JUMP LENGTH PDF}

Equation (5) is a one-sided PDF, as $r \geqslant 0$. It is possible to obtain from Eq. (5) a two-sided PDF, $g(z)$, that refers to the distribution of jumps projected on an arbitrary axis (see the Appendix). This gives the jump length PDF for isotropic homogeneous three-dimensional (3D) space, irrespective of the azimuthal dependence, and is therefore the PDF to be used in a unidimensional representation of radiation migration like the commonly used cases of plane parallel stratified stellar atmospheres or the idealized one-dimensional (1D) geometries in laboratory scale atomic vapor ensembles. ${ }^{11}$ This PDF is

$$
g(z)=\int_{|z|}^{\infty} \frac{f(r)}{2 r} d r=\int_{1}^{\infty} \frac{f(|z| u)}{2 u} d u .
$$

Using Eqs. (5) and (29), the two-sided jump length PDF is obtained as

$$
g(z)=\frac{1}{2} \int_{-\infty}^{\infty} \Phi(u)^{2} E_{1}(|z| \Phi(u)) d u,
$$

where $E_{1}(x)$ is the exponential integral function, defined as $E_{1}(x)=\int_{x}^{\infty} \exp (-u) / u d u=\int_{1}^{\infty} \exp (-x u) / u d u$. It follows from Eq. (29) that for all the cases discussed the asymptotic behavior of $g(z)$ is identical to that of $f(r)$. 


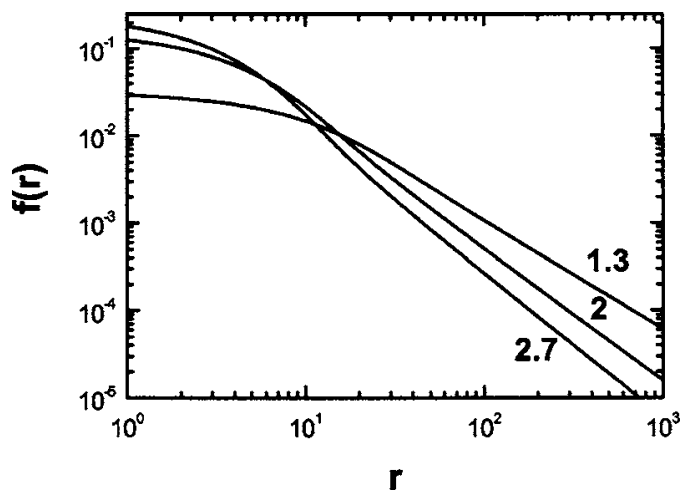

FIG. 1. One-sided jump length PDF [Eq. (5)] for the generalized Lorentz line shape [Eq. (33)] for $p=1.3, p=2$ (Lorentz), and $p=2.7$.

\section{RESULTS FOR SELECTED LINE SHAPES}

For the Lorentz line shape [Eq. (9)] $p=2$, hence $^{8} \mu=\frac{1}{2}$. For the Doppler line shape [Eq. (8)],

$$
\Psi(k)=\sqrt{-\ln (\sqrt{\pi} k)},
$$

and Eq. (18) gives

$$
f(r) \sim r^{-2}(\ln r)^{-1 / 2} \sim r^{-2} .
$$

Therefore $^{8} \mu=1$, as mentioned above.

One may also consider more general line shape functions, in order to better understand the relation between the asymptotic behaviors of $\Phi(x)$ and of $f(r)$. We shall consider a generalized Lorentz line shape,

$$
\Phi_{\mathrm{GL}}(x)=\frac{N}{1+|x|^{p}} \quad(p>1),
$$

where $N$ is a normalization constant,

$$
N=\frac{1}{2 \pi} p \sin (\pi / p)
$$

as well as a generalized Doppler line shape,

$$
\Phi_{\mathrm{GD}}(x)=N \exp \left(-|x|^{\beta}\right) \quad(\beta>0),
$$

with

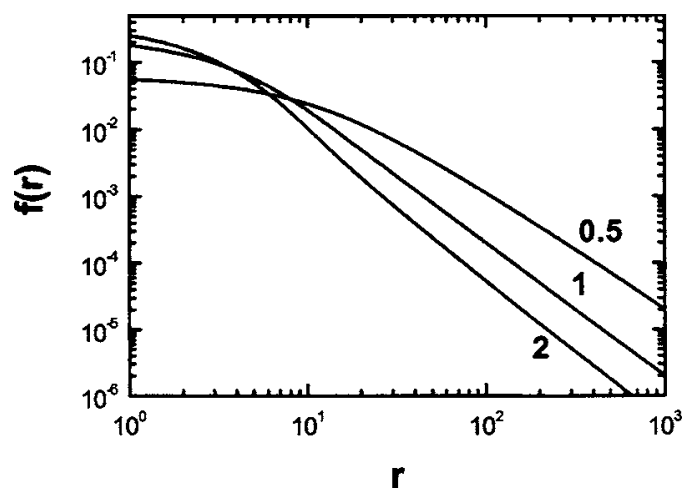

FIG. 2. One-sided jump length PDF [Eq. (5)] for the generalized Doppler line shape [Eq. (35)] for $\beta=0.5$ (stretched exponential), $\beta=1$ (Laplace), and $\beta=2$ (Doppler).

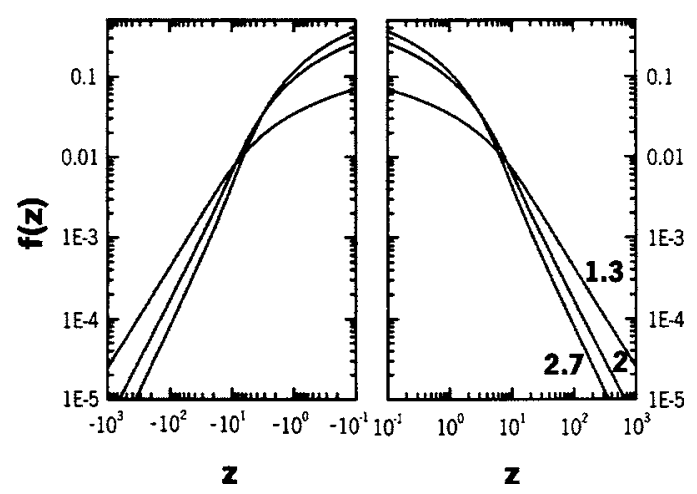

FIG. 3. Two-sided jump length PDF [Eq. (29)] for the generalized Lorentz line shape [Eq. (33)] for $p=1.3, p=2$ (Lorentz), and $p=2.7$.

$$
N=\frac{1}{2 \Gamma(1+1 / \beta)} .
$$

The generalized Doppler line shape [Eq. (35)] encompasses the Laplace distribution $(\beta=1)$, and the symmetric stretched $(\beta<1)$, and compressed $(\beta>1)$ exponentials, as well as the Gaussian distribution $(\beta=2)$.

The one-sided jump length PDFs of the generalized Lorentz and Doppler line shapes are shown in Figs. 1 and 2, respectively, for representative values of the respective parameters. The asymptotic behavior starts to be observed for opacities between 5 and 30, depending on the line shape and on the numeric value of the corresponding parameter.

The two-sided jump length PDFs of the generalized Lorentz and Doppler line shapes are shown in Figs. 3 and 4, respectively, for representative values of the respective parameters. These PDFs are infinite for $z=0$, owing to the significant contribution of jumps almost orthogonal to the $z$ axis (note that this already occurs for an exponential jump length PDF, as discussed in the Appendix).

The opacity distribution functions are

$$
H_{\mathrm{GL}}(k)=\frac{2 N}{p k}\left(\frac{N}{k}-1\right)^{1 / p-1} \quad(0<k<N)
$$

and

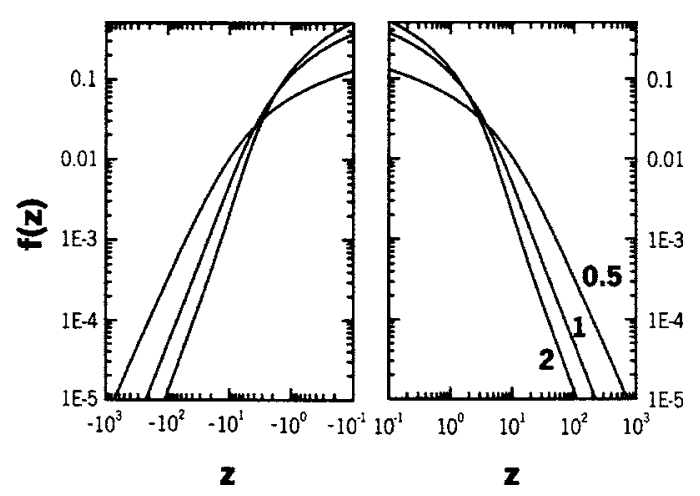

FIG. 4. Two-sided jump length PDF [Eq. (29)] for the generalized Doppler line shape [Eq. (35)] for $\beta=0.5$ (stretched exponential), $\beta=1$ (Laplace), and $\beta=2$ (Doppler). 


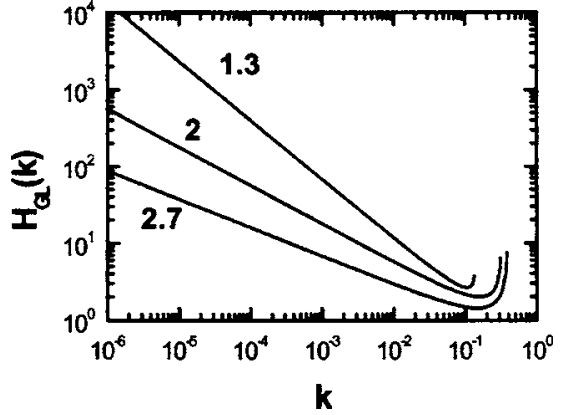

FIG. 5. PDF of effective opacities for the generalized Lorentz line shape [Eq. (37)] for $p=1.3, p=2$ (Lorentz), and $p=2.7$.

$$
H_{\mathrm{GD}}(k)=\frac{2}{\beta}\left[-\ln \left(\frac{k}{N}\right)\right]^{1 / \beta-1} \quad(0<k<N)
$$

(see Figs. 5 and 6). It is apparent that an increase in the parameters $p$ and $\beta$ gives more weight to the higher effective opacities.

Equation (26) applies to the generalized Lorentz line shape, hence $\mu=1-1 / p$, and the most extreme superdiffusive behavior is obtained for $p \rightarrow 1$ (see also Figs. 1, 3, and 5). From Eq. (7), this also implies that $r_{c}(t) \sim t^{1 /(1-1 / p)}$ $\sim t^{1 /(p-1)}$ for $p \rightarrow 1$.

For the generalized Doppler line shape, on the other hand, it can be concluded from Eq. (18) or (21) that $\mu=1$ holds irrespective of the value of $\beta$. One thus arrives to the general result that the lighter the tail of $\Phi(x)$, the less heavy the tail of $f(r)$, although this tail is always heavy, as $\mu \leqslant 1$. The superdiffusive nature of the random flight originates from the fact that whenever the frequency goes into the tail of the spectral distribution, the mean free path increases enormously. In a simulation, the long but infrequent jumps must be generated with precise weights. For this purpose, the use of the effective opacity PDF is preferable to the direct use of line shape functions.

Trajectories generated from the generalized Lorentz and Doppler line shapes by the Monte Carlo method are shown in Figs. 7-9. Figure 7 shows that the overall topology of the points visited by the fractal random walk depends mainly on the shape of the spectral distribution. Within each type, an increase in the value of parameters $\beta$ or $p$ reduces the importance of the larger jumps (see the overall scales and es-

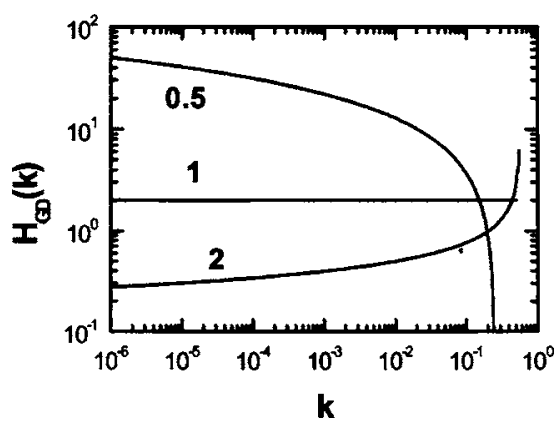

FIG. 6. PDF of effective opacities for the generalized Doppler line shape [Eq. (38)] for $\beta=0.5$ (stretched exponential), $\beta=1$ (Laplace), and $\beta=2$ (Doppler).

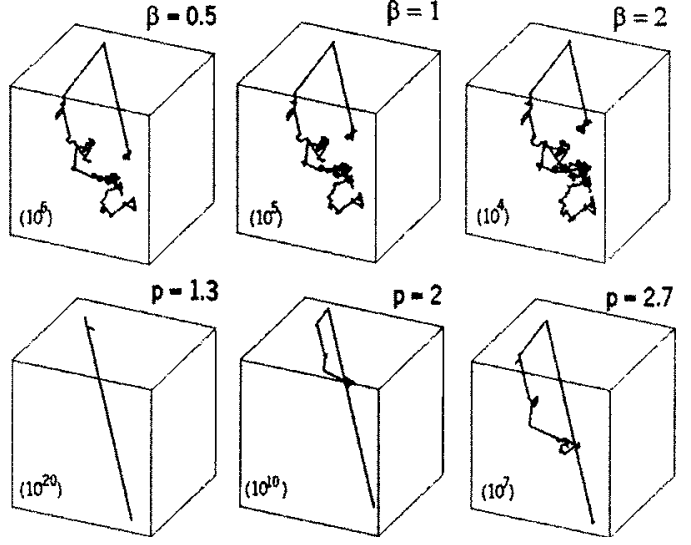

FIG. 7. Single trajectories of 50000 jumps each for the generalized Lorentz and Doppler line shapes in infinite 3D medium (box length in parentheses). All trajectories were obtained with the same random number sequence.

pecially the series of results for the Lorentz distributions). Figures 8 and 9 show the self-similar character of the trajectories.

The Voigt line shape, ${ }^{11,13}$

$$
\Phi_{V}(x)=\frac{a}{\pi^{3 / 2}} \int_{-\infty}^{+\infty} \frac{e^{-u^{2}}}{a^{2}+(x-u)^{2}} d u,
$$

which is the convolution of the Gaussian and Cauchy line shapes, is frequently used to take into account the joint effect of natural broadening (pure Lorentz) or collisional broadening (to a first approximation described by a Lorentzian line shape) and of Doppler broadening. Parameter $a$ measures the relative importance of the Lorentz linewidth with respect to the Doppler linewidth. For $a=0$ the line shape is pure Doppler, while for $a=1$ it is already very close to pure Lorentz in the entire frequency range, as shown in Fig. 10. The Voigt line shape function is known to have Doppler character close to the line center, and to be dominated by the Lorentz function in the wings, provided $a>0$. Therefore, for any nonzero value of parameter $a$, the asymptotic behavior of the Voigt line shape is identical to that of the Lorentz function. The effective opacity PDF for the Voigt line shape was numerically computed and is shown in Fig. 11 for several values of

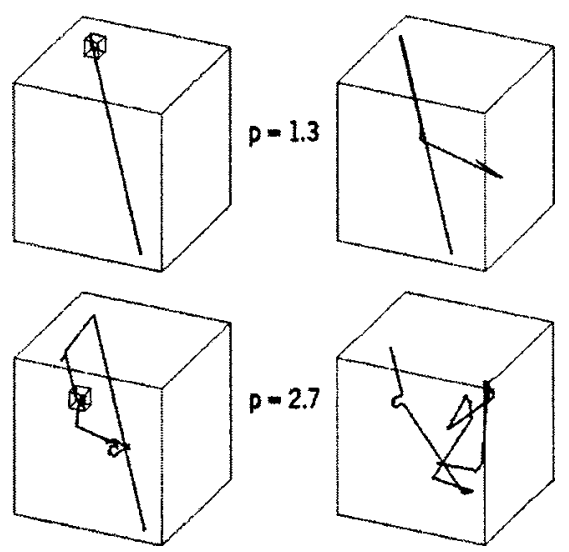

FIG. 8. Single trajectories for the generalized Lorentz line shapes of Fig. 7 displaying self-similar behavior (10\% of the whole scale zoomed on the right). 


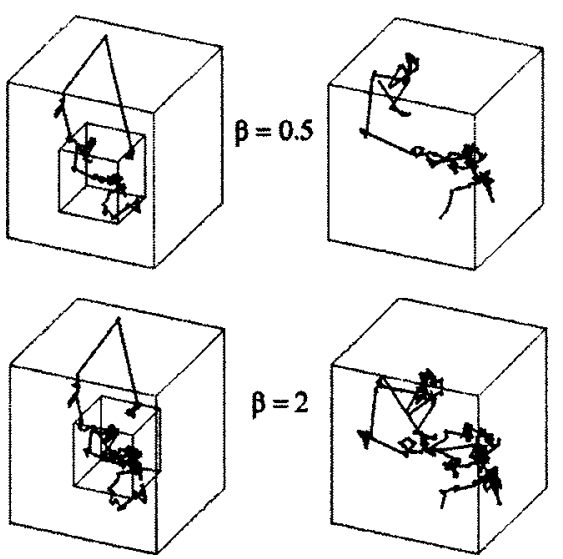

FIG. 9. Single trajectories for the generalized Doppler line shapes of Fig. 7 displaying self-similar behavior $(40 \%$ of the whole scale zoomed on the right).

parameter $a$. It is again observed that for $a=0.5$ it is close to Lorentz's, whereas for $a=10^{-4}$ it coincides with the Doppler PDF for most of the opacities.

\section{CONCLUSIONS}

In this work, we considered the relation between the one-sided jump length PDF and the line shape function in resonance radiation trapping in atomic vapors. General relations that give the one-sided jump length PDF and the Lévy parameter $\mu$ for any line shape were obtained. The two-sided jump length probability density function along an arbitrary axis was also derived. As a side result, a procedure to obtain the Maxwell distribution of velocities from the MaxwellBoltzmann distribution of speeds was obtained. The results were applied to generalized Doppler, generalized Lorentz, and Voigt line shape functions. It was concluded that the lighter the tail of the line shape function, the less heavy the tail of the one-sided jump length PDF, although this tail is always heavy, as $\mu \leqslant 1$. An important theoretical aspect that remains to be tackled is the effect of partial frequency redistribution on the above results. The experimental study of resonance radiation trapping in atomic vapors with an emphasis on its spatial aspects may provide a clear physical example of a superdiffusive Lévy flight.

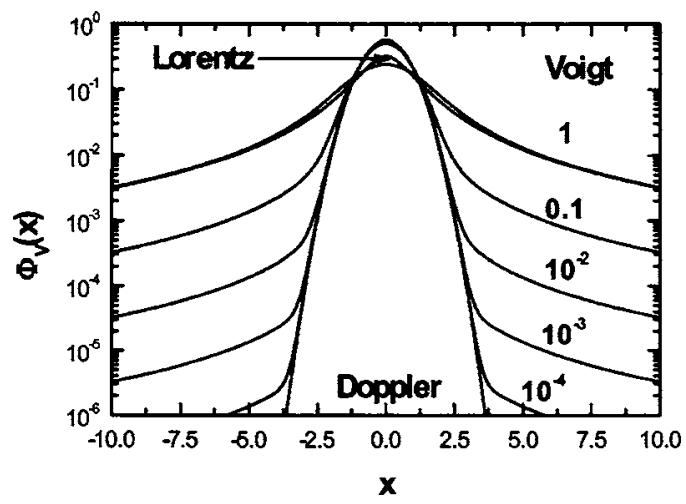

FIG. 10. The Voigt line profile [Eq. (39)] for several values of the $a$ parameter.

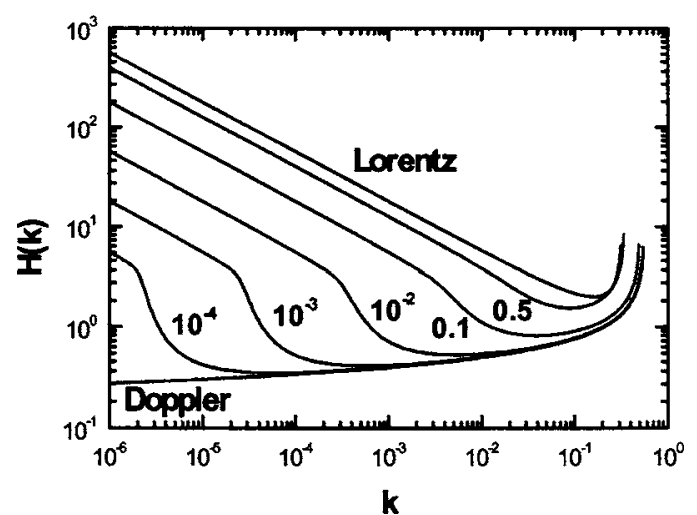

FIG. 11. PDF of effective opacities for the Voigt line shape [Eq. (39)] as a function of the $a$ parameter.

\section{ACKNOWLEDGMENTS}

This work was supported by Fundação para a Ciência e Tecnologia (FCT) (Portugal) under Project No. POCTI/ 58535/QUI/2004 and by FCT and Universidade do Minho (Portugal) under Project No. REEQ/443/EEI/2005.

\section{APPENDIX: CALCULATION OF THE TWO-SIDED JUMP LENGTH PDF}

Without loss of generality, the axis for which the twosided jump length PDF is computed may be conveniently taken to be the $z$ axis. In spherical coordinates, $z=r \cos \theta$. Given the assumption of isotropy, the PDFs of $r$ and of $\theta$ are, respectively, $f(r)$ and $1 / 2 \sin \theta$. Considering at first only non-negative values of $z$ and using the random variable transformation theorem, ${ }^{1}$ the PDF for $z$ is given by

$$
\begin{aligned}
g(z) & =\int_{0}^{\infty} \int_{0}^{\pi / 2} \int_{0}^{2 \pi} \delta(z-r \cos \theta) \frac{1}{4 \pi} \sin \theta f(r) d \varphi d \theta d r \\
& =\frac{1}{2} \int_{0}^{\infty} \int_{0}^{\pi / 2} \delta(z-r \cos \theta) \sin \theta f(r) d \theta d r
\end{aligned}
$$

or, making the change of variable $u=\cos \theta$,

$$
g(z)=\frac{1}{2} \int_{0}^{\infty} \int_{0}^{1} \delta(z-u r) f(r) d u d r,
$$

that can in turn be transformed into

$$
\begin{aligned}
g(z) & =\frac{1}{2} \int_{0}^{\infty} \frac{f(r)}{r}\left(\int_{0}^{r} \delta(v-z) d v\right) d r \\
& =\frac{1}{2} \int_{0}^{\infty} \delta(v-z)\left(\int_{v}^{\infty} \frac{f(r)}{r} d r\right) d v,
\end{aligned}
$$

hence it is obtained that

$$
g(z)=\int_{z}^{\infty} \frac{f(r)}{2 r} d r \quad(z \geqslant 0) .
$$

Given the symmetry of the problem, the final result, valid for any $z$, is 


$$
g(z)=\int_{|z|}^{\infty} \frac{f(r)}{2 r} d r=\int_{1}^{\infty} \frac{f(|z| u)}{2 u} d u
$$

A side comment is appropriate at this point: a well-known procedure in the elementary kinetic theory of gases is the calculation of the Maxwell-Boltzmann distribution of speeds from the Maxwell distribution of velocities. ${ }^{14}$ Mutatis mutandis, Eq. (A5) allows to do the opposite, i.e., to obtain the Maxwell distribution of velocities from the MaxwellBoltzmann distribution of speeds.

The simplest case that can be considered for application of Eq. (A5) is an isotropic random walk in 3D with constant step length, $f(r)=\delta(r-l)$. It is then obtained that $g(z)$ $=1 /(2 l)$ for $-l<z<l$ and $g(z)=0$ otherwise. Another case of interest is that of an exponential distribution of step lengths, $f(r)=r_{0}^{-1} \exp \left(-r / r_{0}\right)$ where $r_{0}$ is the average jump length. For this PDF one obtains $g(z)=\left(2 r_{0}\right)^{-1} E_{1}\left(|z| / r_{0}\right)$, where $E_{1}(x)$ is the exponential integral function, defined as $E_{1}(x)$ $=\int_{x}^{\infty} \exp (-u) / u d u=\int_{1}^{\infty} \exp (-x u) / u d u$.
${ }^{1}$ N. G. van Kampen, Stochastic Processes in Physics and Chemistry, 2nd ed. (North-Holland, Amsterdam, 1992).

${ }^{2}$ J.-P. Bouchaud and A. Georges, Phys. Rep. 4\&5, 127 (1990).

${ }^{3}$ Lévy Flights and Related Topics in Physics, edited by M. F. Shlesinger, U. Frisch, and G. M. Zaslavsky (Springer, New York, 1994).

${ }^{4}$ R. Metzler and J. Klafter, Phys. Rep. 339, 1 (2000).

${ }^{5}$ R. Metzler and J. Klafter, J. Phys. A 37, R161 (2004).

${ }^{6}$ D. Brockmann, L. Hufnagel, and T. Geisel, Nature (London) 439, 462 (2006)

${ }^{7}$ E. J. N. Pereira, M. N. Berberan-Santos, and J. M. G. Martinho, J. Chem. Phys. 104, 8950 (1996).

${ }^{8}$ E. Pereira, J. M. G. Martinho, and M. N. Berberan-Santos, Phys. Rev. Lett. 93, 120201 (2004).

${ }^{9}$ A. Gallagher, in Atomic, Molecular, and Optical Physics Handbook, edited by G. W. F. Drake (AIP, New York, 1996).

${ }^{10}$ M. N. Berberan-Santos, E. J. N. Pereira, and J. M. G. Martinho, in Resonance Energy Transfer, edited by D. L. Andrews and A. A. Demidov (Wiley, Chichester, 1999).

${ }^{11}$ A. F. Molisch and B. P. Oehry, Radiation Trapping in Atomic Vapours (Oxford University Press, Oxford, 1998).

${ }^{12}$ M. N. Berberan-Santos, J. Math. Chem. 38, 165 (2005).

${ }^{13}$ A. Thorne, U. Litzén, and S. Johansson, Spectrophysics (Springer, Berlin, 1999).

${ }^{14}$ C. E. Hecht, Statistical Thermodynamics and Kinetic Theory (Dover, New York, 1998). 